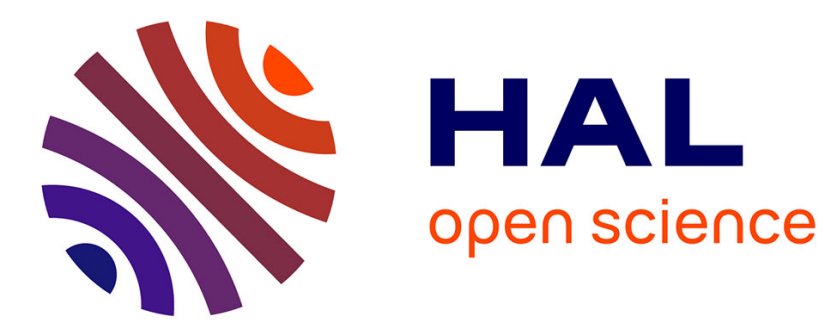

\title{
Inter Screen, Between Humans and Avatars
}

Etienne Armand Amato, Claire Sistach, Lucile Haute

\section{To cite this version:}

Etienne Armand Amato, Claire Sistach, Lucile Haute. Inter Screen, Between Humans and Avatars. VRIC, Apr 2012, Laval, France. hal-02012768

\section{HAL Id: hal-02012768 https://hal.science/hal-02012768}

Submitted on 9 Feb 2019

HAL is a multi-disciplinary open access archive for the deposit and dissemination of scientific research documents, whether they are published or not. The documents may come from teaching and research institutions in France or abroad, or from public or private research centers.
L'archive ouverte pluridisciplinaire HAL, est destinée au dépôt et à la diffusion de documents scientifiques de niveau recherche, publiés ou non, émanant des établissements d'enseignement et de recherche français ou étrangers, des laboratoires publics ou privés. 


\section{“INTER SCREEN”, BETWEEN HUMANS AND AVATARS}

\author{
AMATO Etienne-Armand \\ Gobelins - UP8 - OMNSH \\ eamato@gmail.com
}

\author{
SISTACH Claire \\ Artiste indépendante \\ claire.sistach@gmail.com
}

\author{
HAUTE Lucile \\ EnsadLab/EN-ER - CIEREC \\ lucile.haute@gmail.com
}

\begin{abstract}
Que se passe-t-il quand une installation interactive convie des avatars et de simples êtres humains pour induire reconnaissance et compréhension mutuelles ? Inter Screen a tenté cette expérience auprès d'un large public. Le propos de cet article est d'expliciter ce dispositif inhabituel et facile d'accès, et de présenter les premiers résultats obtenus. De la sorte, il met en lumière la singularité des rapports intersubjectifs impliquant des avatars dans un contexte artistique et scientifique contrôlés.
\end{abstract}

\section{Keywords}

Avatar, Art \& Science, Dispositif, Univers simulé, Représentation

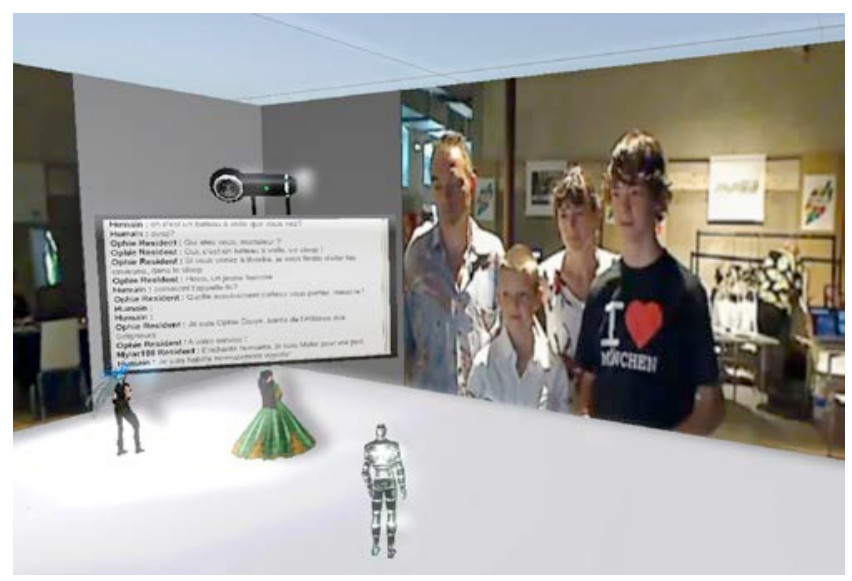

\section{UN DISPOSITIF ENTRE LES MONDES}

Présenté en juin 2011 au Centquatre, centre d'art parisien, à l'occasion du festival Futur en Seine, Inter Screen a été réalisé par Claire Sistach, artiste numérique, Etienne-Armand Amato, chercheur en communication et Lucile Haute, chercheuse en esthétique [1]. Cette installation interactive construit un dispositif de communication reliant de façon originale notre monde physique et un univers en ligne pour mettre en présence avatars $3 \mathrm{D}$ et êtres humains. Elle a permis au public d'expérimenter une situation d'interaction inédite, à savoir rencontrer directement des avatars, sans avoir besoin d'en utiliser un lui-même.

Comment? Les visiteurs du lieu culturel étaient filmés par une webcam dont le flux vidéo était retransmis en temps réel dans un espace 3D, sur la plateforme Second Life. Ils devenaient ainsi visibles pour ses habitants numériques. Le public assistait donc à la réunion de personnages $3 \mathrm{D}$ à l'écran, tout en voyant sa propre image, d'où la possibilité d'une communication gestuelle expressive à base de signes et de mimiques. De plus, une console de messagerie instantanée externe et dédiée (avec un clavier et un second écran) servait au dialogue entre les avatars présents sur la Sim [2] et les humains. De la sorte, le visiteur se trouvait confronté à des avatars issus d'univers tels que Second Life, WoW ou Eve Online. Ils avaient accepté de parler en leur nom propre, en tant que sujet constitué par l'historique de leur vie numérique en ligne.

D'un point de vue théorique, Inter Screen se définit à la fois comme une œuvre esthétique et comme un moyen d'étude. Cette proposition constitue donc une "recherche-action artistique" [3], ici appliquée aux sciences de l'information et de la communication, visant à provoquer, décrire et analyser les rapports avec les avatars. Le dispositif lui-même a construit un terrain d'étude pour observer les comportements singuliers qu'il favorisait. En parallèle, pour servir de référence, une enquête en ligne a servi à interroger les relations habituelles que les joueurs/utilisateurs entretiennent avec leur(s) avatar(s) 3D.

\section{DE L'IDENTITE DES AVATARS}

En informatique, le terme avatar désigne un personnage représentant un utilisateur sur Internet et dans les jeux vidéo. Or celui-ci peut avoir diverses formes et fonctions : une représentation prédéterminée des actions d'un joueur sous la forme d'un protagoniste préconstruit (un héros de jeu vidéo comme Mario), une fiche de profil d'un réseau social, ou encore une représentation interactive créée par l'utilisateur.

Inter Screen s'est concentré sur cette dernière catégorie, réputée susciter une émergence identitaire forte. En effet, le contexte des univers 3D persistants et multi-utilisateurs [4] forge parfois une personnalité complexe et sociale particulière qui s'autonomise de celle du joueur. Pour convier plusieurs de ces individualités numériques, un "appel à avatars" [5] a été lancé sur Internet, qui s'adressait aux avatars eux-mêmes, non à leur opérateur. Il a permis de rassembler des profils distincts, correspondant à plusieurs critères combinables :

- Identité imaginaire (distincte de la vie de son créateur) versus extension virtuelle de l'utilisateur ;

- Avatar natif de Second Life versus «métavatars » (ou méta-avatar, c'est-à-dire des avatars d'avatar) issus d'autres univers et venus endosser un nouveau corps synthétique sur ce métavers pour l'occasion.

Parmi ces «métavatars», distinguons d'un côté les migrants investissant les mondes numériques à leur gré car ils se sont affranchis de leur univers d'origine et tout contexte de jeu particulier, et de l'autre, ceux restant liés à leur MMORPG. Ces derniers étaient donc toujours dépendants de leur environnement de roleplay. Pour les faire participer à Inter Screen, il a fallu élaborer une narration cohérente avec leur fiction initiale.

Ces personnalités numériques ayant accepté de s'impliquer dans Inter Screen ont surpris les visiteurs du Centquatre. Les «humains" se demandaient s'ils avaient affaire à des intelligences artificielles (bot), à des pseudo-individualités ou encore, à des acteurs s'amusant de leur incrédulité. La mise en scène et en écran des altérités constituées a brouillé les frontières entre fiction et réalité. En a résulté des réactions diverses : de 
l'impolitesse ouverte des visiteurs à l'égard des avatars, à de longs échanges philosophiques, poétiques ou humoristiques, en passant par des conversations plus banales ou conventionnelles.

\section{AU-DELA DU DUALISME DE L'ECRAN}

Le paradigme de l'écran [6] scinde le monde en deux parties exclusives l'une de l'autre: le champ et le hors-champ, le tangible et l'immatériel, la réalité et les représentations. Cette césure irréconciliable semble constitutive des espaces 3D simulés: l'espace physique du joueur/utilisateur s'oppose à celui des représentations numériques, que celles-ci renvoient à un imaginaire ou à un mimétisme documentaire. Il y aurait un pas infranchissable de l'un à l'autre, chacun se targuant de qualités propres.

Sur la bien-nommée plateforme Second Life, le ballet des avatars semble autiste à toute interaction avec l'espace tangible. Evoluant simultanément entre deux environnements, apparait un sujet hybride qui serait piégé entre l'espace 3D présumé « non-réel », et l'espace devant l'ordinateur, aux horizons limités. Inter Screen propose de mettre en crise cette dialectique pour instaurer une expérience identitaire tout autre.

En occultant complètement la relation entre l'avatar et son joueur/utilisateur, Inter Screen propose au public d'interroger son propre rapport à la représentation interactive. Simultanément, l'avatar est mis en valeur en tant que sujet unique et complexe, hybride de matérialités et temporalités distinctes. L'avatar est abordé comme une construction identitaire pleine et entière, une matérialisation et expression de l'imaginaire dans un contexte artificiel donné, contraint par les principes algorithmiques de la computation et de la connexion qui régissent son existence

La réconciliation de deux univers habituellement étrangers l'un à l'autre, s'opère grâce à la relation intersubjective construite par le dispositif Inter Screen. D'une part, le devenir-image des visiteurs induit par leur captation vidéo fait écho aux corps interactifs constituant la partie visible des avatars. Par ailleurs, l'interaction gestuelle en temps réel entre deux publics trouve une généalogie dans les travaux de Kit Galloway et Sherrie Rabinovitz, en particulier Hole In Space [7]. Cette installation participative mettait en relation des passants dans l'espace public, par des écrans, haut-parleurs, système de captation vidéo et sonore, présentés dans deux vitrines de magasins, l'un à New York, l'autre à Los Angeles. Comment faire rencontre, événement commun, tandis que nous nous tenons à distance ? Telle est l'une des interrogations qui sous-tend cette proposition. Inter Screen déporte la question : il ne s'agit plus de mettre en rapport deux espaces physiquement distants (réponse au fantasme d'ubiquité) mais d'articuler deux espaces à la matérialité et temporalité distinctes se télescopant pour produire un entre-deux monde intermédiaire.

\section{DE L'INTERACTION DES SUJETS}

La relation aux personnages $3 \mathrm{D}$ peut évoquer la notion latine de persona. Personnifier, c'est parler à travers le masque, jouer un rôle, assurer la représentation, au tribunal aussi bien qu'au théâtre. À la «réalité » de l'individu se substitue la "virtualité » de la personne [8]. Inter Screen, occulte ce prérequis et arrière-plan identitaire, au seul bénéfice d'une rencontre avec une personnalité complexe, riche d'une histoire et d'un imaginaire singuliers, que seule la conversation mettait à jour. Nombre de visiteurs se demandèrent, notamment : «Qu'est-ce qui anime ces représentations anthropomorphes 3D? Quel est leur hors- champ et causalité : humain ou automate?» En réponse, les médiateurs [9] se contentaient de mentionner que des avatars avaient répondu à l'appel qui leur avait été fait de rencontrer des humains. Ce faisant, ils les invitaient à résoudre leurs interrogations en investissant, par leur participation, le dispositif.

Dans un tel cadre performatif, au sens linguistique d'Austin, l'avatar apparaissait comme sujet agissant unitaire plutôt que comme manifestation seconde d'un sujet (le joueur/utilisateur) manipulant une représentation objectivée. Il se faisait puissance vivante, et non actualisation d'actions programmées ou interprétation d'un scénario prédéfini.

Dans le même temps, le visiteur avec l'avatar se trouvait renvoyé à sa propre persona. Le phénomène se concrétise par la qualité spéculaire du dispositif qui s'affirme dans l'intégration, à l'écran, de l'image vidéo filmant un participant se voyant en miroir aux côtés des avatars. La représentation de chacun se fait performative, tandis que s'opère un dépassement du dualisme constitutif de l'écran: la dissociation "réel / imaginaire » se déporte vers celle «tangible / représentation». Le virtuel, l'artificiel, le fictif produisent du réel à partir de l'apparence [10] et de l'interaction. Une hybridation des sujets est à l'œuvre, conjointe à la traversée de l'écran promise [11].

Les technologies de communication déployées dans les univers simulés semblent offrir à l'humain qui les emploie un "être » et un «devenir» nouveaux et singuliers, au-delà de tous les possibles connus auparavant. En produisant cette confrontation, mise en miroir et dialogue de l' «être» humain avec sa part métamorphosée par la technologie, la pertinence d'Inter Screen est de faire resurgir directement et d'interroger autrement les fondamentaux existentiels de notre propre condition.

\section{REFERENCES}

[1] Hébergement sur Second Life: La Bibliothèque Francophone du Metaverse - Partenaires: Association Coalition Cyborg, EnsadLab/EN-ER, ICAN, OMNSH, CITU-Paragraphe Contributions: Alain Barthélémy (développement informatique), Frederick Thompson (build 3D)

[2] Terme générique désignant une région de l'espace simulé.

[3] Terme établi par Etienne Armand Amato pour désigner une recherche-action, qui consiste en une méthode de recherche scientifique fondée par Kurt Lewin, dont le terrain d'action et de prospection est celui de l'expérimentation artistique. Cf. C. Sistach, "Inter Screen, une recherche-action artistique », 2012, artsciencefactory : $\quad \mathrm{http} / / /$ artsciencefactory.fr/2012/02/14/interscreen-une-recherche-action-artistique/

[4] Il s'agit soit de métavers tel que Second Life ou OpenSim, soit de MMORPG (Massively Multiplayer Online Role Playing Games)

[5] http://inter-screen.blogspot.com/p/avatars-recherches.html

[6] Sur la notion d'écran chez Roland Barthes et son adaptation aux nouvelles technologies, voir: Lev Manovitch, Le langage des nouveaux Medias, Paris, les presses du réel, 2010, pp.214-224 (MIT Press, 2001)

[7] Hole In Space, Kit Galloway et Sherrie Rabinovitz, liaison satellitaire NY-LA, 1980.

[8] Cf. Hobbes, Leviathan, trad. Tricaud, ed. Sirey, 1983, pp. 161, 62-63

[9] Tom Giraud, Vincent Levy, Romain Pechot, Aurélia Raoull

[10] Christiane VOLLAIRE, «De Hobbes à Foucault, La virtualité du corps politique réalisée dans le corps biologique », colloque Retour au virtuel : vie et cultures numériques, cnam, février 2012

[11] Amato Etienne Armand, Le jeu vidéo comme dispositif d'instanciation, thèse de doctorat en Sciences de l'Information et de la Communication, Université Paris 8, 2008. 